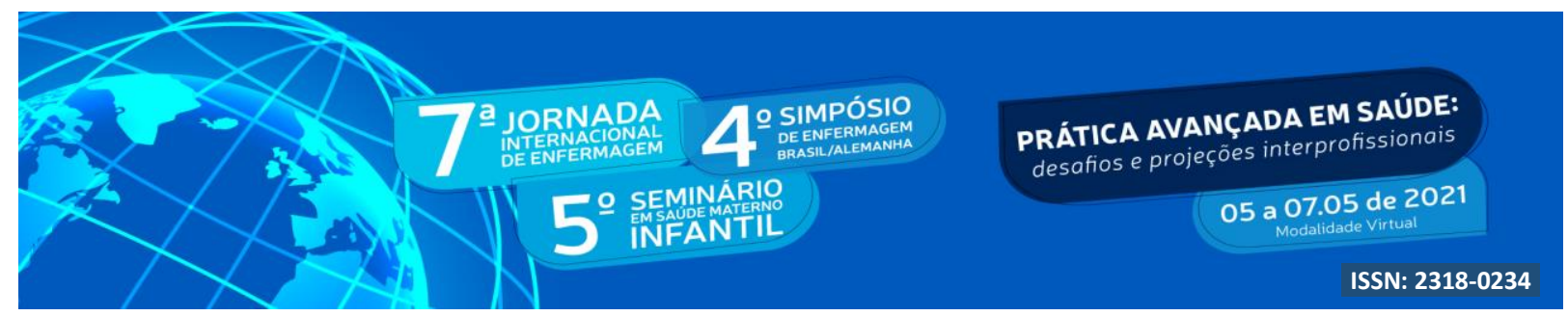

DOI: http://doi.org/10.48195/jie2021-162

\title{
VISITA ÀVIGILÂNCIA EM SAÚDE DO TRABALHADOR: RELATO DE EXPERIÊNCIA
}

\section{Francisco Fernandes'; Frederico Bigolin Ferraz²; Jhonata Carpes³; Juliana Silveira Colomé $^{4}$}

\section{RESUMO}

O presente estudo objetiva relatar a experiência de atividades de visita a $4^{\circ}$ Coordenadoria Regional de Saúde de Santa Maria - RS $\left(4^{\circ} \mathrm{CRS}\right)$. Trata-se de um relato de experiência realizado no decorrer de um dia de visitação pela disciplina de Políticas Publicas em Saúde na $4^{\circ}$ Coordenadoria Regional de Saúde na área da Vigilância em Saúde do Trabalhador localizada no centro de Santa Maria, Rio Grande do Sul. As atividades foram desenvolvidas no primeiro semestre de 2018 por três acadêmicos do curso de Enfermagem da Universidade Franciscana. A visita proporcionou aos acadêmicos uma visão mais clara e ampla dos processos em saúde com foco no trabalhador, ampliando suas visões quanto ao sistema público de saúde.

Palavras-chave: Saúde do Trabalhador; Políticas Públicas; Sistema Público.

\begin{abstract}
This study aims to report the experience of visiting activities to the 4th Regional Health Coordination of Santa Maria - RS (4th CRS). This is an experience report made during a day of visitation by the Public Health Policies discipline at the 4th Regional Health Coordination in the area of Occupational Health Surveillance located in the center of Santa Maria, Rio Grande do Sul. Activities were developed in the first semester of 2018 by three academics from the Nursing course at the Franciscan University. The visit provided academics with a clearer and broader view of health processes with a focus on workers, expanding their views on the public health system.
\end{abstract}

Key Words: Worker's health; Public policy; Public System.

\footnotetext{
${ }^{1}$ Acadêmico de Graduação em Enfermagem - UFN 01franciscofernandes@gmail.com

2 Acadêmico de Graduação em Enfermagem - UFN jhonatacarpes@ hotmail.com

${ }^{3}$ Acadêmico de Graduação em Enfermagem - UFN fredferraz14@gmail.com

${ }^{4}$ Orientadora. Doutora em Enfermagem. Docente da Universidade Franciscana - UFN juliana@ufn.edu.br
} 


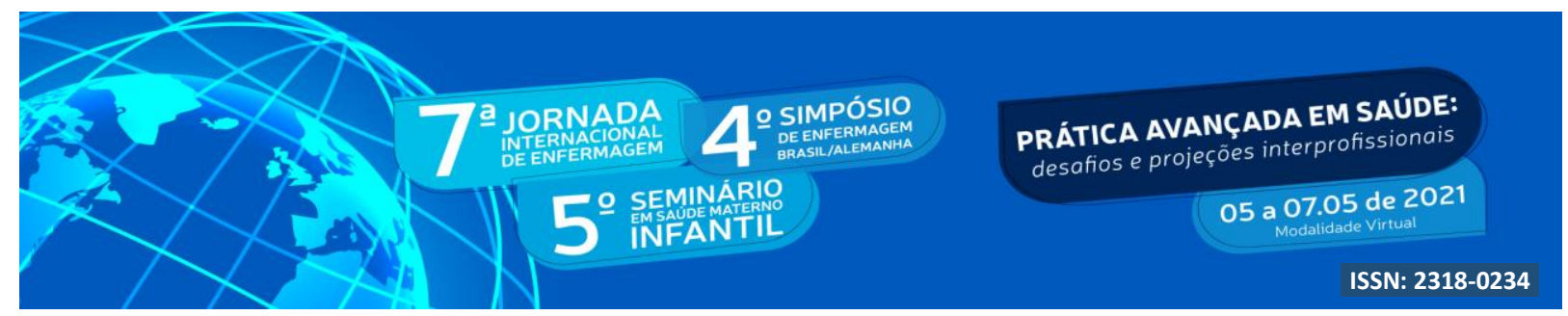

\section{INTRODUÇÃ̃O}

O relato de experiência se traduz em texto acadêmico descrevendo precisamente uma dada experiência que possa contribuir de forma relevante para uma área de atuação profissional. Caracteriza-se por uma vivência profissional considerada importante para a formação dos futuros profissionais da área da enfermagem (SOLDATELI, MARTINS, 2018).

A Constituição Federal de 1988 incorporou as questões de Saúde do Trabalhador ao enunciar o conceito ampliado de Saúde, incluindo entre seus determinantes as condições de alimentação, habitação, educação, renda, meio ambiente, trabalho, emprego e ao atribuir ao SUS à responsabilidade de coordenar as ações no país. (DIAS; HOEFEL; 2005)

Em agosto do ano de 2012 o Ministério da Saúde por meio da portaria $n^{\circ} 1.823$ instituiu a política nacional de saúde do trabalhador e da trabalhadora que veio para regulamentar e nortear as ações em saúde do trabalho.

A Vigilância em Saúde do Trabalhador (VST) vem se firmando como uma área de atuação da saúde pública e, nesse sentido, necessita atentar para essas mudanças em curso nos processos de saúde/doença e na atuação da saúde pública em geral, a fim de poder dar respostas efetivas aos problemas colocados para os trabalhadores - problemas estes de ordem bastante complexa e de difícil resolução mediante unicamente ações curativas e preventivas. (ALVES; 2003)

Para o encaminhamento e efetividade de ações de promoção da saúde, é defendida a participação da comunidade, a VST necessita invariavelmente estabelecer parcerias, principalmente com os trabalhadores, que são os maiores interessados. (ALVES; 2003)

A Saúde do Trabalhador (ST) necessita considerar que saúde dos trabalhadores não se remete apenas a problemas diretamente relacionados a aspectos do processo de trabalho no qual se insere e atuar sobre ela. Isso não significa dizer que a Vigilância em Saúde do Trabalhador (VST) deva dar conta de todos esses fatores e agir sobre eles, e sim que deve procurar estabelecer parcerias, por meio da intersetorialidade, a fim de buscar uma atuação mais abrangente e eficaz. (ALVES; 2003)

\section{OBJETIVO}




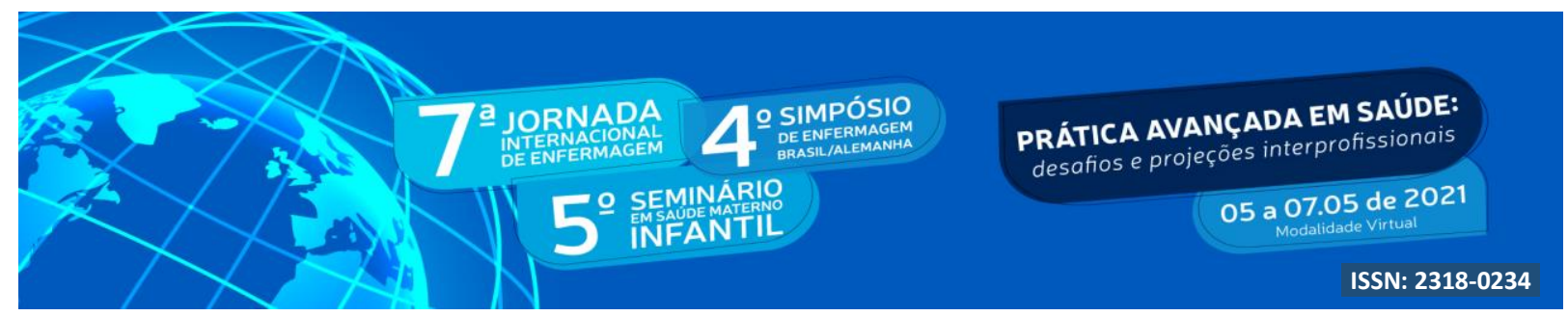

O presente estudo tem como objetivo abordar a experiência vivenciada por acadêmicos de enfermagem diante o serviço prestado pela $4^{\circ} \mathrm{CRS}$ e descrever sobre sua competência diante os municípios que são dependentes do referente órgão.

\section{METODOLOGIA}

Trata-se de um relato de experiência desenvolvido no decorrer das aulas teóricas praticas da disciplina de Políticas Publicas em Saúde. A $4^{\circ}$ CRS está localizada na região central de Santa Maria - RS.

As atividades de visita foram realizadas no primeiro semestre de 2018, por três acadêmicos do $4^{\circ}$ semestre de Enfermagem da UFN. A visita realizada contou como aula normal da disciplina e foram acompanhadas pela responsável pela vigilância em saúde do trabalhador.

A visita a $4^{\circ} \mathrm{CRS}$ foi realizada em apenas um momento do semestre. A visita foi realizada já com a divisão dos grupos feita pelos alunos, onde cada grupos escolhia um tema que gostaria de realizar o trabalho.

Foi realizada apenas uma visita com duração de 3 horas. Salienta-se que a visita foi realizada com dia e horário decididos previamente, com anotações feitas com autorização da responsável.

\section{RESULTADOS E DISCUSSÃO}

A visita foi realizada por conta da cadeira realizada na Universidade Franciscana ser relacionada as políticas públicas em saúde onde é um campo essencial para a organização do Sistema Único de Saúde (SUS) e para a vigilância dos diversos campos que a saúde se compõe.

O trabalho realizado por este serviço é diverso, como a análise de casos de acidentes do trabalho e também na utilização da paramentação dos profissionais da saúde, conhecidos como Equipamentos de Proteção Individual (EPI,s). Este serviço contém como apoio os demais, tal como o Cerest (Centro de referência em saude do trabalhador). Os CEREST têm por objetivo organizar as funções para dispor de suporte técnico, de educação permanente, de 


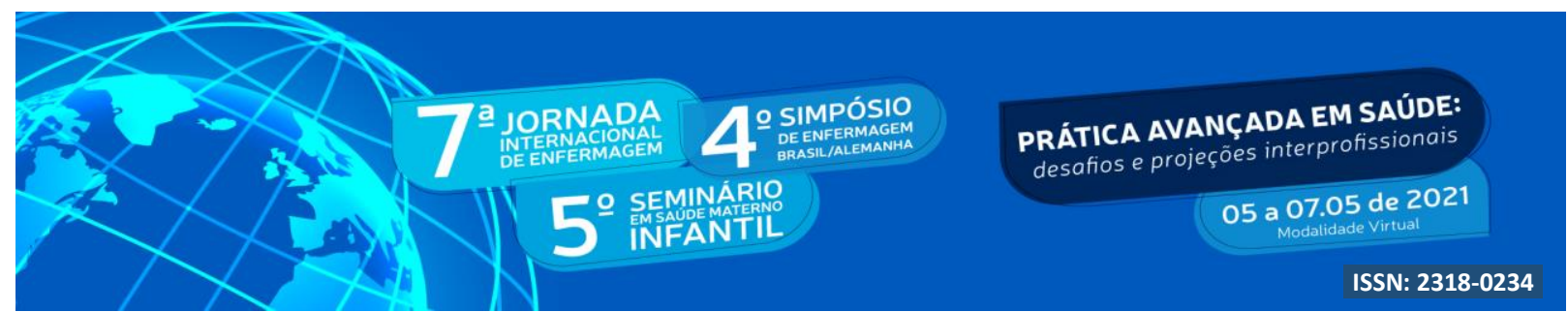

coordenação de planos de promoção, proteção e vigilãncia, além de permitir a utilização da referência e contrarrefêrencia para a rede de assitência à saúde dos trabalhadores (SOLDATELI, MARTINS, 2018).

\subsection{Depoimentos}

4.1.1 - A visita feita na $4^{\circ}$ coordenadoria regional de saúde foi muito interessante, pois não imaginava que o SUS era tão amplo e organizado para ter grupos de gestores que estão atuando em áreas específicas, como a saúde do trabalhador que seria o foco meu e do meu grupo nesta visita onde foi explicado de perto como funcionava o serviço e que me deixou impressionado pela quantidade de pessoas indo atrás de evidências e pesquisas epidemiológicas para diminuir o índice de diferentes tipos de patologias e agravos sociais. $\mathrm{O}$ que deixou claro de como as pessoas julgam o Sistema Único de Saúde (SUS), onde todos têm acesso e com direitos e benefícios exclusivos para diversos pontos, sendo amparado por profissionais capacitados a lidarem com situações extremas e resolver problemas únicos de determinadas comunidades, garantindo um saneamento básico adequado e um cuidado integral a saúde humana. Além de termos sido bem recepcionados por pessoas especializadas em diversas áreas, me atenderam com muita atenção, onde forneceram lembranças e café da manhã.

4.1.2 - A visita a $4^{\circ} \mathrm{CRS}$ foi uma experiência com um caráter relevante para o grupo onde nos ampliou a visão de como funciona o SUS e como em sua tese é o melhor e mais completo plano de saúde pública existente. Percebeu-se que os funcionários eram empenhados em realizar o seu trabalho da melhor forma possível apesar das dificuldades que o sistema público oferece. Buscam otimizar os recursos disponíveis e fazer ações de seu dever mesmo com o baixo auxílio financeiro. $\mathrm{Na}$ única visita que fizemos fomos muito bem recepcionados, foram atenciosos conosco, sanaram todas nossas duvidas, explicaram como funciona a Vigilância em Saúde do Trabalhador, as delimitações de área que é dividida em duas, Verdes Campos e Entre Rios, os encaminhamentos das UBS para o CEREST (Centro de Referência a Saúde do Trabalhador), a distribuição de renda, materiais e pacientes em todo o 


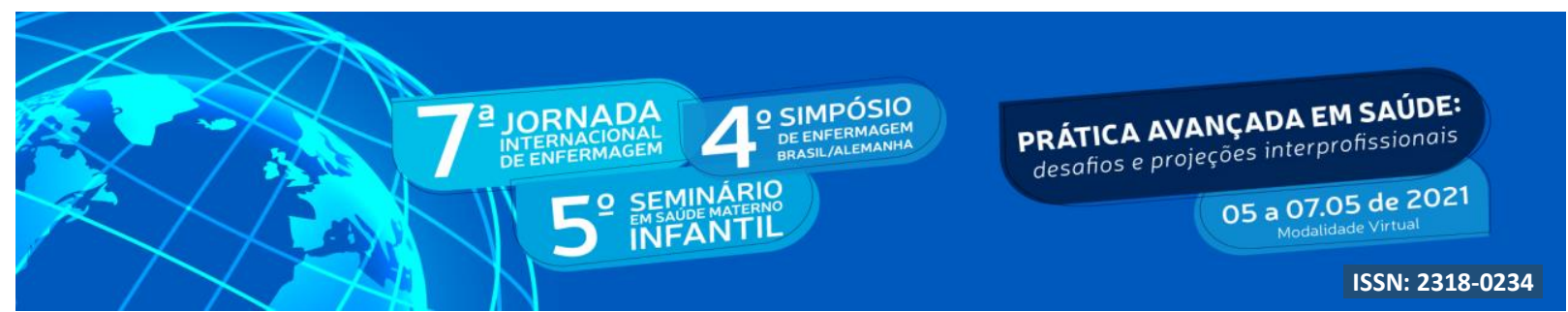

território da $4^{\circ} \mathrm{CRS}$, até nos deram materiais de campanhas da saúde juntamente com um café, pois era cedo da manhã e já estávamos lá e foram solidários conosco.

4.1.3 - A ida até a Vigilância em Saúde do Trabalhador (Visat) agregou bastante ao conhecimento de como funciona a rede de saúde no município. Diversas vigilâncias se encontram no prédio da $4^{\circ} \mathrm{CRS}$ onde todas atuam de forma interligadas, compartilhando informações e planejando estratégias e ações em conjunto. Entender sobre o que se trata a Visat e como ela funciona, regulamentando, inspecionando e supervisionando as atividades trabalhistas na região. Agindo em ações e campanhas de prevenção de acidentes e promoção de saúde no ambiente de trabalho. Em principal atuação no interior onde há grande concentração de trabalhadores na agricultura onde o cuidado é redobrado até por conta do uso de insumos em plantações e o não uso de EPI's nesses locais. Ficou evidenciado que o trabalho na Visat é constante e está sempre em evolução e possui o intuito de prever uma maneira segura de condições de trabalho e saúde.

\section{CONCLUSÃO}

As visitas a $4^{\circ}$ Coordenadoria Regional de Saúde propiciou aos acadêmicos uma nova visão melhorada do que é SUS e como ele funciona. Conseguiu se ver como cada funcionário é empenhado em fazer o seu trabalho da maneira mais correta, e que apesar do que a população diz, o SUS funciona, é o melhor plano de saúde pública existente e busca atender os usuários da melhor forma para diminuir as demandas sociais atribuidas no cotidiano do Brasil e também o sobrecarregamento dos profissionais da saúde inseridos em todos os serviços disponibilizados pelo amparo à saúde que o país proporciona.

Segundo Vianna et.al (2017), a Visat (Vigilância em saúde do trabalhador) exerce um papel fundamental para a garantia da saúde do trabalhador nos municípios atuantes em parceria com o CEREST, onde ambos atuam em caráter de promoção a saúde e prevenção de acidentes. 


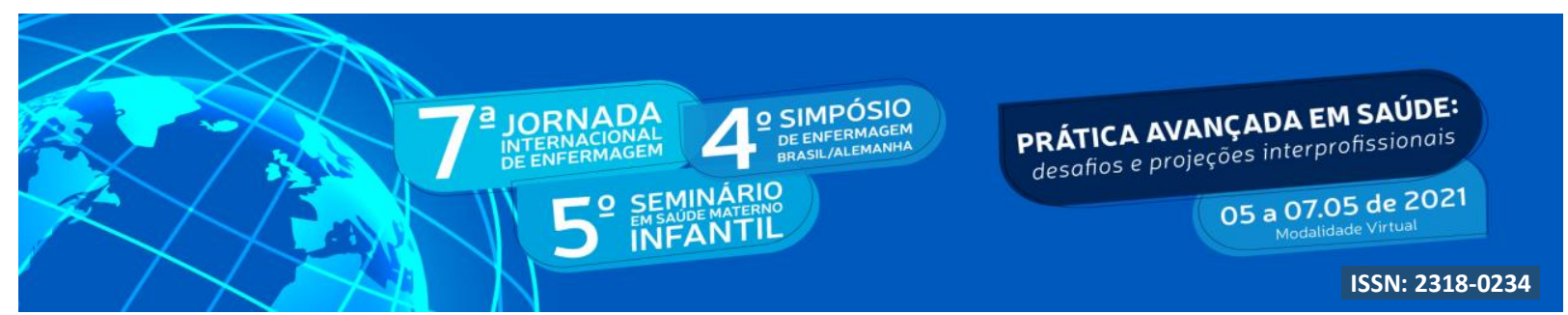

\section{REFERÊNCIAS}

DIAS, E.C., HOEFEL, M, da G. O desafio de implementar as ações de saúde do trabalhador no SUS: a estratégia da RENAST. Rio de Janeiro. Ciência \& Saude Coletiva. Vol. 10, $\mathrm{n}^{\circ} 4$, pág. 817- 828, setembro, 2005.

ALVES, R.B. Vigilância em saúde do trabalhador e promoção da saúde: aproximações possíveis e desafios. Cad. Saúde Pública, Rio de Janeiro, vol.19, n º1, pág, 319-322, jan-fev, 2003.

VIANNA, L. C. R, et al. Vigilância em Saúde do Trabalhador: um estudo à luz da Portaria ${ }^{\circ}$ 3.120/98. Saúde em Debate. Vol. 41, nº 114, pág. 786-800. Jul-set 2017.

SOLDATELI, T; MARTINS, R. V. O papel do CEREST na saúde do trabalhador: uma revisão narrativa. Manancial, repositório digital da UFSM, Encantado RS, Pág. 1-23, abril, 2018. 